\title{
Association between cerebral dopamine neurotrophic factor (CDNF) 2 polymorphisms and schizophrenia susceptibility and symptoms in the Han Chinese population
}

Yongfeng Yang 1,2,3, Hongyan Yu², Wenqiang Li ${ }^{2,3}$, Bing Liư ${ }^{4}$, Hongxing Zhang ${ }^{2,3}$, Shuang Ding ${ }^{2,3}$, Yanli Lü ${ }^{2,3}$, Tianzi Jiang ${ }^{1,4,5,6^{*}}$ and Luxian $\mathrm{LV}^{2,3,7^{*}}$

\begin{abstract}
Background: Schizophrenia (SZ) is a complex polygenic psychiatric disorder caused in part by abnormal dopamine levels. Cerebral dopamine neurotrophic factor (CDNF) 2 is known to protect and repair the dopaminergic system. Dopamine dysfunction is one of the pathogenesis of SZ. However, the relationship between CDNF2 and SZ has not been previously investigated. We speculated that CDNF2 may be a susceptibility factor for SZ.

Methods: To address this issue, we carried out a study to investigate the association between CDNF2 and SZ in the total sample 1371 (670 SZ patients and 701 healthy controls) Han Chinese population. Stage 1 included 528 SZ patients and 528 healthy controls; and stage 2 included 142 SZ patients and 173 healthy controls. The allele and genotype frequencies of five single nucleotide polymorphisms (rs2577074, rs2577075, rs2249810, rs6506891, and rs2118343) of CDNF2 were compared between patients and controls.

Results: We found a significant association in allele and genotype frequencies between the two groups at rs 2249810 $\left(x^{2}=4.38\right.$ and 6.45 , respectively; $P=0.03$ and 0.04 , respectively). An association was also observed in males at rs2249810 ( $X^{2}=8.76 ; P=0.03$ ). Haplotype TGATC differed between $S Z$ and controls in stage 2 samples $\left(X^{2}=6.38\right.$; $P=0.01)$, and rs2118343 genotypes were associated with negative factor scores $(F=4.396 ; P=0.01)$.

Conclusions: These results suggest that rs2249810 and haplotype TGATC of CDNF2 are an SZ susceptibility locus and factor, respectively, and that rs2118343 genotypes are associated with negative symptoms of SZ in the Han Chinese population.
\end{abstract}

Keywords: CDNF2, Polymorphism, PANSS, Schizophrenia

\section{Background}

Schizophrenia (SZ) is a complex psychiatric disorder with an estimated heritability of $60-80 \%$ that affects $1.0 \%$ of the global population [1-3]. Previous studies have reported that the absolute risk of suicide in SZ to be $6.55 \%$ in male and $4.91 \%$ in female [4], and suicide

*Correspondence: jiangtz@n|pria.ac.cn; Ivx928@126.com

${ }^{5}$ National Laboratory of Pattern Recognition, Institute of Automation, Chinese Academy of Sciences, Beijing 100190, China

7 Department of Psychiatry of the Second Affiliated Hospital of Xinxiang Medical University, No. 388, Jianshe Middle Road, Xinxiang 453002, China Full list of author information is available at the end of the article was 13-fold higher for SZ than the general population [5]. The core symptoms of SZ vary in terms of severity among patients [6]. Research on the genetic basis of SZ has focused on identifying polymorphisms in candidate genes and linkage regions [7-10]. To date, 108 SZ-associated gene loci have been identified in a genome-wide association analysis (GWAS) of the largest sample size to date $(36,989$ cases vs. 113,075 controls) [11]. However, others have reported that multiple genes contribute weakly or moderately to SZ pathogenesis [12]. Identifying SZ susceptibility genes among numerous candidates is an ongoing challenge. 
Abnormal dopamine levels underlie SZ. Neurotrophic factors, such as brain-derived neurotrophic factor $(B D N F)$, nerve growth factor, glial cell line-derived neurotrophic factor, and cerebral dopamine neurotrophic factor $(C D N F)$, can repair and protect the dopaminergic system. Previous studies have shown that $B D N F$ is involved in the pathophysiology of SZ, with $B D N F$ levels reduced in the serum and cerebrospinal fluid of patients [13]. CDNF has been shown to protect and repair the dopaminergic system in rat models of Parkinson's disease (PD) [14], which is characterized by degeneration of dopaminergic neurons; it may thus have therapeutic benefits [15]. However, the relationship between $C D N F$ and $\mathrm{SZ}$ has not been previously investigated. We speculated that $C D N F$ is a susceptibility factor for SZ. We tested this hypothesis by examining CDNF single nucleotide polymorphisms (SNPs) in two study stages with independent sample sets.

\section{Methods}

Stage 1 samples were those from our previous publications [16, 17], and included 528 paranoid SZ patients (mean age: $27.32 \pm 8.03$ years old) and 528 healthy controls (mean age: $27.73 \pm 8.01$ years old) who were recruited from March 2005 to December 2008. Stage 2 samples included $142 \mathrm{SZ}$ patients (paranoid, $\mathrm{n}=122$ and undifferentiated, $\mathrm{n}=20$; mean age: $29.28 \pm 7.17$ years old) and 173 healthy controls (mean age: $32.49 \pm 7.43$ years old) who were recruited from May 2011 to December 2014.

SZ was diagnosed as previously described $[16,17]$ according to the criteria listed in the Diagnostic and Statistical Manual of Mental Disorders Fourth Edition (DSM-IV). Exclusion criteria were as follows: patients had been diagnosed with other psychiatric disorders; or had organic brain disease, substance dependence, severe medical complications, or neurological diseases. Family mental health history (FH) was defined as at least one first- or second-degree relative of the proband who met DSM-IV criteria for SZ or schizoaffective disorder. The Positive and Negative Symptom Scale (PANSS) was used to evaluate psychotic syndromes. Five factors were derived from the PANSS, including positive symptoms, negative symptoms, cognition, expression/anxiety, and excitement/hostility [18].

A total of $372 \mathrm{SZ}$ patients (stage $1, \mathrm{n}=229$ and stage 2 , $\mathrm{n}=143$ ) who were not taking antipsychotic medications were evaluated for psychotic syndromes using the PANSS [19]. Inclusion and exclusion criteria for healthy controls were as described our previous papers [16, 17]. These subjects were screened by psychiatrists in simple nonstructured interviews. All participants were unrelated
Han Chinese who were born and living in North Henan province.

Five SNPs were selected as described in our previous work $[16,17]$ and covered the 26974145-26995906 genomic intron region on chromosome 10. In stage 1, genotyping was carried out as detailed in our earlier studies [16, 17] using Illumina GoldenGate assays on a BeadStation 500G Genotyping System (Illumina, San Diego, CA, USA). In stage 2, samples were genotyped using a standard Illumina genotyping protocol.

Statistical analyses in this study were performed as described in our previous papers $[16,17]$. Power analyses were performed using the G*Power software to calculated (http://www.gpower.hhu.de/) for this study $[20,21]$. Genotype and allele frequencies were analyzed using Haploview v.4.1. Hardy-Weinberg equilibrium was assessed with the $\chi^{2}$ test with one degree of freedom. Associations between the five factors from the PANSS and different genotype carriers were evaluated by analysis of variance. Bonferroni correction for multiple pairwise comparisons was conducted for the $\mathrm{X} \times$ phenotype interaction to reduce the probability of false positives. $P<0.05$ was considered statistically significant. The corrected $\alpha^{\prime}(\mathrm{P}=0.01)$ is $\alpha(\mathrm{P}=0.05)$ divided by the number of possible comparisons.

\section{Results}

To identify allelic variants of the CDNF2 gene that are associated with SZ, we analyzed two sets of samples. The sampling success rate for subjects and SNPs was 99.84\%. Power analyses revealed that the total sample size $(\mathrm{n}=1371)$ had a power of 0.96 to detect a small effect $(r=0.1-0.23)$, and a power of 1.00 to detect both medium $(r=0.24-0.36)$ and large $(r>0.37)$ effects on genotype distributions. For allele frequency, the sample size $(n=2742)$ had the power $(0.91-1.00)$ to detect small, medium, and large effects.

None of the genotype distributions of the other four SNPs significantly deviated from HWE except for SNP rs6506891. There were no significant differences in genotype and allele frequencies between SZ and controls at five SNPs in stage 1 samples $(P>0.05$, Table 1$)$, even after subdividing by gender and FH $(P>0.05)$. However, there were significant differences in genotype and allele frequencies between SZ and controls at rs2249810, rs6506891, and rs2118343 in stage 2 samples. We also noted differences at rs2249810 when stage 1 and 2 samples were combined. This association was present at rs2249810 in males $\left(\chi^{2}=8.76 ; P=0.03\right)$. To further analyze haplotype structures in this sample, we evaluated pairwise linkage disequilibrium (LD) of five SNPs in SZ patients and controls using standardized $\mathrm{D}^{\prime}$ and $\mathrm{r}^{2}$ values. Haplotypes were identified at five SNPs of CDNF2 


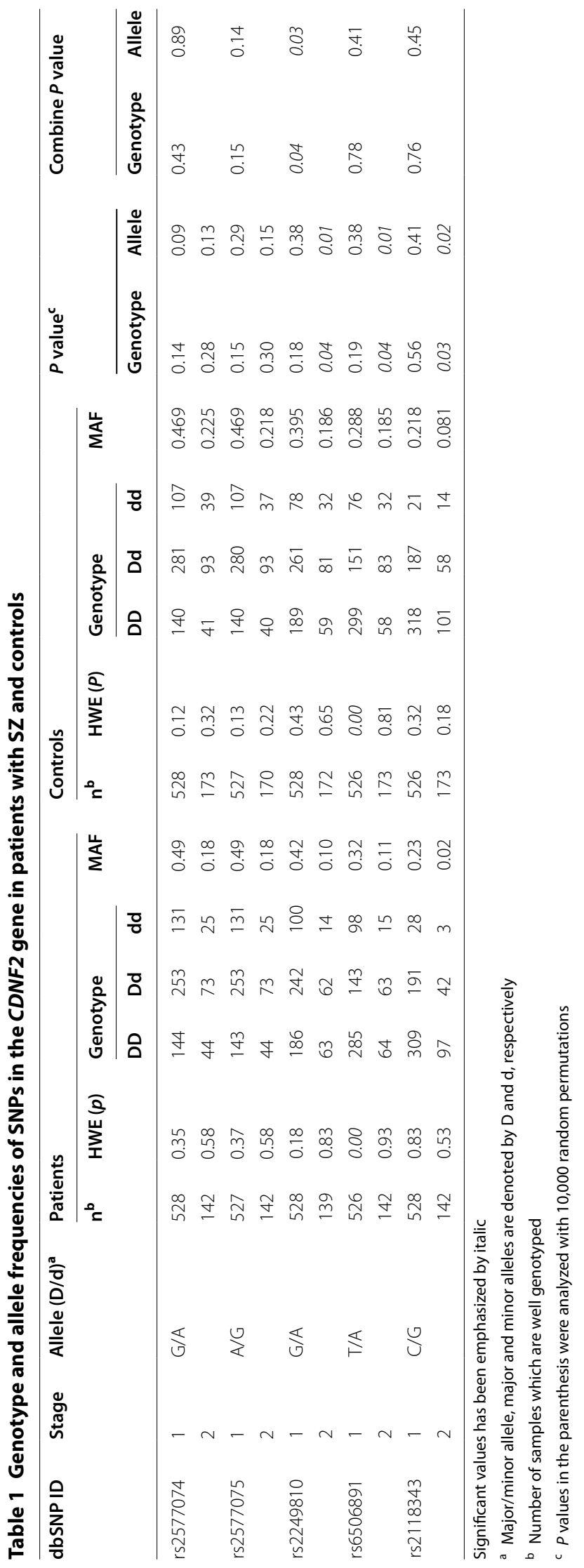


in stage 1 and 2 samples. The positions of these SNPs in stage 2 , LD structure, and $\mathrm{D}^{\prime}$ values for all variants are shown in Fig. 1. Five SNPs formed one LD block, yielding four haplotypes; one of these (TGATC) differed significantly between $\mathrm{SZ}$ and controls only in stage 2 samples $\left(X^{2}=6.38 ; P=0.01\right)$ (Table 2$)$.

To explore the association between CDNF2 variations and SZ symptoms, 371 first-onset SZ patients (stage $1, \mathrm{n}=228$ and stage $2, \mathrm{n}=143$ ) with complete PANSS scores were selected from stages 1 and 2. Only rs2577075 genotypes were associated with cognition factor scores in stage 2 samples after Bonferroni correction ( $\mathrm{F}=3.39 ; P=0.03$ ) (Table 3 ). We also only found rs2577075 genotypes were associated with cognition factor scores in female when subdivided by gender after

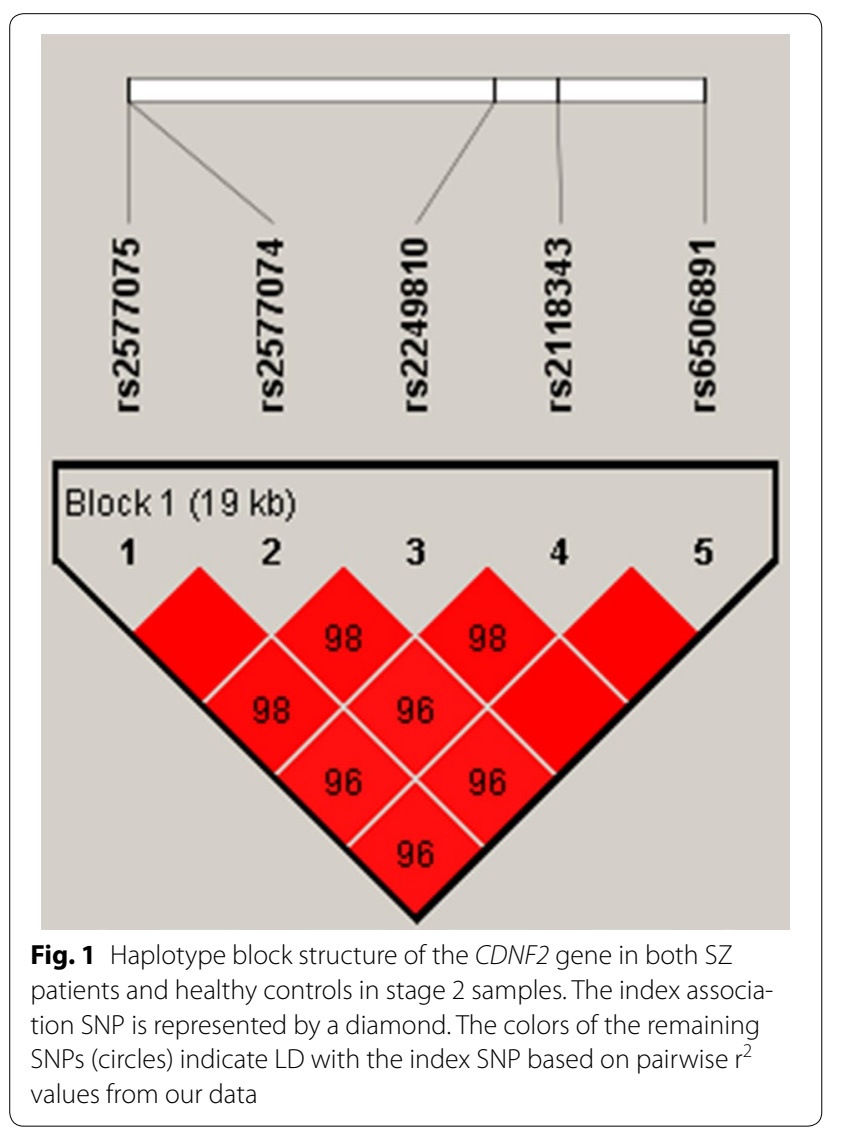

Bonferroni correction $(\mathrm{F}=4.05 ; P=0.04)$. Other SNPs from stage 1 and 2 samples did not show any associations $(P>0.05)$. However, rs2118343 genotypes were significantly associated with negative factor scores when stage 1 and 2 samples were combined $(\mathrm{F}=4.396, P=0.01)$. We found rs2118343 genotypes were associated with depression/anxiety and excitement/hostility scores in female when subdivided by gender in combined samples after Bonferroni correction ( $\mathrm{F}=4.39,4.73 ; P=0.04,0.03$, respectively).

\section{Discussion}

This study investigated CDNF2 mutations associated with $\mathrm{SZ}$ and associated symptoms in the Han Chinese population. Significant differences were found in genotype and allele frequencies of rs2249810 between SZ patients and healthy controls, suggesting that $C D N F 2$ is a susceptibility gene for SZ. We also found that rs2118343 genotypes are associated with negative psychotic symptoms of SZ.

$C D N F$ has strong neuroprotective and restorative effects in animal models of PD [22], and protects dopaminergic neurons in the 6-hydroxydopamine rat model $[14,23]$. However, the genotype and allele frequencies of the CDNF SNPs rs1901650 and rs11259365 did not differ between PD patients and controls; only the $C$ allele of $C D N F$ rs7094179, an intronic SNP, has been linked to PD susceptibility [24, 25].

Intron variant was relation to the $C D N F 2$ gene organization. $C D N F 2$ can protect and repair the dopaminergic system, and may thus have an important role in PD [14, $26,27]$. Five SNPs of $C D N F 2$ in our study were located in intron region of chromosome 10 , which variation may lead to dopaminergic system disfunction. Meanwhile, multiple SZ susceptibility loci have been found on chromosome 10 [7, 8]. In stage 1 samples, we did not detect any associations in paranoid SZ patients, possibly because we did not examine other SZ subtypes. We therefore included the undifferentiated subtype in the stage 2 analysis, and found that rs 2249810 of CDNF2 may be a susceptibility locus in SZ.

$\mathrm{SZ}$ is influenced by dopamine, glutamate, and serotonin neurotransmission systems. We previously reported associations between Solute carrier family 6 member 4

Table 2 Haplotypes of CDNF2 in stage 2 patients with SZ

\begin{tabular}{lcccr}
\hline Haplotype & Case (freq) & Control (freq) & Chi square & Odds ratio (95\% CI) \\
\hline CAGAG & $157.87(0.57)$ & $169.89(0.50)$ & 2.25 & 0.13 \\
TGATC & $45.87(0.17)$ & $82.00(0.24)$ & 6.38 & 0.01 \\
TGATG & $43.00(0.16)$ & $57.90(0.17)$ & 0.30 & 0.58 \\
TGGAG & $30.13(0.11)$ & $26.11(0.08)$ & 2.61 & $0.61(0.41-0.92)$ \\
\hline
\end{tabular}

Significant value has been emphasized by italic

$\mathrm{Cl}$ confidence interval 


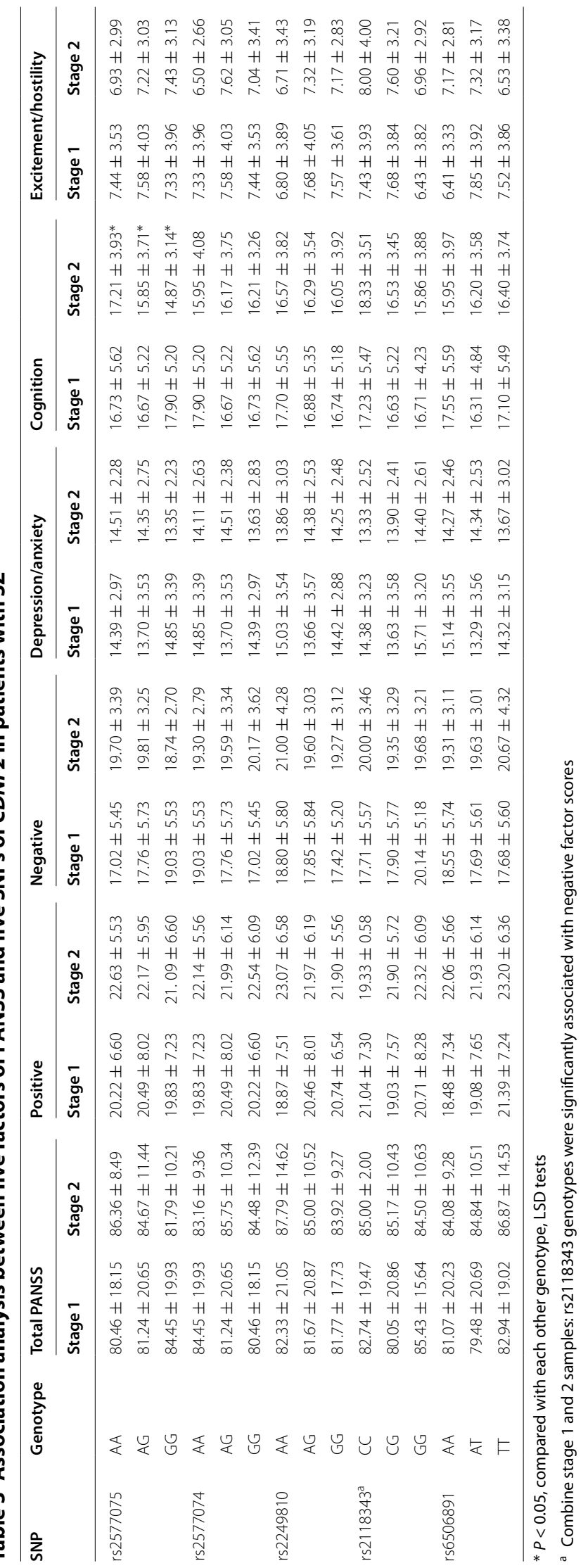


[17] in the serotoninergic system and Glutamate ionotropic receptor NMDA type subunit 2B [16] and Reelin [28] in the glutamatergic system and SZ. The present study focused on CDNF2 because of its role in the dopaminergic system. Our results from two sets of samples suggest that SNP rs2249810 of CDNF2 may be a susceptibility locus for $\mathrm{SZ}$ and provide evidence that it is caused by the interaction of a large number of susceptibility genes [29]. To date, there has been a lot of GWAS researches on the susceptibility site of SZ [7-11,30], and a recent meta-analysis using GWAS data also found 30 new sites [31]. However, there is still no reported association between CDNF2 and SZ. The difference between GWAS and our research is samples selected more than three groups and multi-ethnic in the former, one group and only Han Chinese people in the latter. Therefore, our sample has a single genetic background. Meanwhile, we found rs 2577075 genotypes and haplotype TGATC may be susceptibility factors of SZ in stage 2 samples, but those results were not found in total samples. That may be the majority of the samples were paranoid, and with less undifferentiated in total samples. Therefore, those results need to be further validated in a large sample, especially in the samples with multiple subtypes of SZ.

$\mathrm{SZ}$ is characterized by positive and negative symptoms, cognitive deficits, and disorganization of thoughts and behaviors [32, 33]. SZ is considered a dopamine disorder based on the psychosis-inducing effects of dopaminereleasing drugs such as amphetamines, and the antipsychotic effects of drugs that block the dopamine D2 receptor [34]. Dopamine dysfunction has been identified as the major cause of SZ [35]. Our results have shown that $C D N F 2$ gene polymorphisms underlie the manifestations of SZ symptoms; moreover, rs2118343 genotypes were associated with negative factor subscores in SZ patients; this provides the first evidence of an association between CDNF2 and negative symptoms in SZ and is consistent with our previous observations of a genetic basis for SZ symptoms [16, 17, 28].

This study had some limitations. Firstly, our sample size was not large enough to obtain complete PANSS scores. Secondly, although we validated our results in two independent datasets, undifferentiated subtypes were only included in the stage 2 analysis.

\section{Conclusion}

In summary, our findings suggest that SNP rs2249810 of CDNF2 is a novel susceptibility locus in SZ. Additional studies are needed to determine whether there are other SNPs associated with specific SZ subtypes (catatonic, collapse, and residual) and ethnic populations.

\section{Abbreviations}

SZ: schizophrenia; CDNF: cerebral dopamine neurotrophic factor; BDNF: brainderived neurotrophic factor; PD: Parkinson's disease; SNPs: single nucleotide polymorphisms; DSM-IV: diagnostic and statistical manual of mental disorders fourth edition; OR: odds ratio; 95\% Cl: 95\% confidence intervals; LD: linkage disequilibrium; ANOVA: analysis of variance; PANSS: positive and negative symptom scale.

\section{Authors' contributions}

$L L$ and JT participated in the design of the study and made final approval of the version to be published. YY, WL and BL were involved in drafting the manuscript and data analysis. WL, YY, and $\mathrm{HZ}$ carried out the molecular genetic examination. YY, HY, SD and YL conducted sample selection and data management. All authors read and approved the final manuscript.

\section{Author details}

${ }^{1}$ Key Laboratory for Neurolnformation of Ministry of Education, School of Life Science and Technology, University of Electronic Science and Technology of China, Chengdu, China. ${ }^{2}$ Department of Psychiatry, Henan Mental Hospital, The Second Affiliated Hospital of Xinxiang Medical University, Xinxiang, China. ${ }^{3}$ Henan Key Lab of Biological Psychiatry, Xinxiang Medical University, Xinxiang, China. ${ }^{4}$ Brainnetome Center, Institute of Automation, Chinese Academy of Sciences, Beijing 100190, China. ${ }^{5}$ National Laboratory of Pattern Recognition, Institute of Automation, Chinese Academy of Sciences, Beijing 100190, China. ${ }^{6}$ The Queensland Brain Institute, University of Queensland, Brisbane, QLD 4072, Australia. ${ }^{7}$ Department of Psychiatry of the Second Affiliated Hospital of Xinxiang Medical University, No. 388, Jianshe Middle Road, Xinxiang 453002 , China.

\section{Acknowledgements}

The authors thank the patient, their families, and the healthy volunteers for their participation, and the physicians who collect clinical data and blood samples in the Second Affiliated Hospital of Xinxiang Medical University.

\section{Competing interests \\ The authors declare that they have no competing interests.}

\section{Availability of data and materials}

The datasets used and/or analysed during the current study are available from the corresponding author on reasonable request.

\section{Consent for publication}

Not applicable.

\section{Ethics approval and consent to participate}

The study protocol was in accordance with principles of the Declaration of Helsinki and approved by the Ethics Committee of the Second Affiliated Hospital of Xinxiang Medical University. Written, informed consent obtained from each participant after they were informed of the purpose and procedures of the study. All authors had no access to information that could identify individual participants during or after data collection.

\section{Funding}

This work was supported by the National Natural Science Foundation of China (81371472 and U1404811), the National Key Basic Research and Development Program (973) (Grant 2011CB707800), the Medical Science and Technology Foundation of Henan Province (201702131), the Open Project Program of the National Laboratory of Pattern Recognition (NLPR) (201600010).

\section{Publisher's Note}

Springer Nature remains neutral with regard to jurisdictional claims in published maps and institutional affiliations.

Received: 9 October 2017 Accepted: 22 December 2017

Published online: 03 January 2018 


\section{References}

1. Schultz SK, Andreasen NC. Schizophrenia. Lancet. 1999:353:1425-30.

2. Kendler KS, Eaves LJ. Psychiatric genetics (Review of Psychiatry). Washington: American Psychiatric Publishing; 2005.

3. Lichtenstein P, Yip BH, Björk C, Pawitan Y, Cannon TD, Sullivan PF, et al. Common genetic determinants of schizophrenia and bipolar disorder in Swedish families: a population-based study. Lancet. 2009:373:234-9.

4. Nordentoft M, Mortensen PB, Pedersen CB. Absolute risk of suicide after first hospital contact in mental disorder. Arch Gen Psychiatry. 2011;68(10):1058-64.

5. Saha S, Chant D, McGrath J. A systematic review of mortality in schizophrenia: is the differential mortality gap worsening over time? Arch Gen Psychiatry. 2007;64(10):1123-31.

6. Owen MJ, Craddock N, O'Donovan MC. Schizophrenia: genes at last? Trends Genet. 2005;21:518-25.

7. Ripke S, O'Dushlaine C, Chambert K, Moran JL, Kähler AK, Akterin S, et al. Genome-wide association analysis identifies 13 new risk loci for schizophrenia. Nat Genet. 2013:45:1150-9.

8. Hamshere ML, Walters JT, Smith R, Richards AL, Green E, Grozeva D, et al Genome-wide significant associations in schizophrenia to ITIH3/4, CACNA1C and SDCCAG8, and extensive replication of associations reported by the Schizophrenia PGC. Mol Psychiatry. 2013;18:708-12.

9. Yue WH, Wang HF, Sun LD, Tang FL, Liu ZH, Zhang HX, et al. Genomewide association study identifies a susceptibility locus for schizophrenia in Han Chinese at 11p11.2. Nat Genet. 2011;43:1228-31.

10. Shi Y, Li Z, Xu Q, Wang T, Li T, Shen J, et al. Common variants on $8 p 12$ and 1q24.2 confer risk of schizophrenia. Nat Genet. 2011:43:1224-7.

11. Schizophrenia Working Group of the Psychiatric Genomics Consortium. Biological insights from 108 schizophrenia-associated genetic loci. Nature. 2014;511:421-7.

12. McGuffin P, Owen MJ, Farmer AE. Genetic basis of schizophrenia. Lancet 1995;346:678-82

13. Fernandes BS, Berk M, Turck CW, Steiner J, Gonçalves CA. Decreased peripheral brain-derived neurotrophic factor levels are a biomarker of disease activity in major psychiatric disorders: a comparative meta-analysis. Mol Psychiatry. 2014;19:750-1.

14. Lindholm P, Voutilainen MH, Laurén J, Peränen J, Leppänen VM, Andressoo JO, et al. Novel neurotrophic factor CDNF protects and rescues midbrain dopamine neurons in vivo. Nature. 2007;448:73-7.

15. Tang T, Li Y, Jiao Q, Du X, Jiang H, et al. Cerebral dopamine neurotrophic factor: a potential therapeutic agent for Parkinson's disease. Neurosci Bull. 2017. https://doi.org/10.1007/s12264-017-0123-4.

16. Yang $Y$, Li W, Zhang $H$, Yang G, Wang $X$, Ding $M$, et al. Association study of N-methyl-D-aspartate receptor subunit 2B (GRIN2B) polymorphisms and schizophrenia symptoms in the Chinese-Han population. PLoS ONE. 2015;10:e0125925.

17. Li W, Yang Y, Lin J, Wang S, Zhao J, Yang G, et al. Association of serotonin transporter gene (SLC6A4) polymorphisms with schizophrenia susceptibility and symptoms in a Chinese-Han population. Prog Neuropsychopharmacol Biol Psychiatry. 2013;44:290-5.
18. Kim JH, Kim SY, Lee J, Oh KJ, Kim YB, Cho ZH. Evaluation of the factor structure of symptoms in patients with schizophrenia. Psychiatry Res. 2012:197:285-9.

19. Kay SR, Fiszbein A, Opler LA. The positive and negative syndrome scale (PANSS) for schizophrenia. Schizophr Bull. 1987;13:261-76.

20. Faul F, Erdfelder E, Lang A-G, Buchner A. G*Power 3: a flexible statistical power analysis program for the social, behavioral, and biomedical sciences. Behav Res Methods. 2007;39:175-91.

21. Faul F, Erdfelder E, Buchner A, Lang A-G. Statistical power analyses using G*Power 3.1: tests for correlation and regression analyses. Behav Res Methods. 2009:41:1149-60.

22. Kemppainen S, Lindholm P, Galli E, Lahtinen HM, Koivisto H, Hämäläinen $\mathrm{E}$, et al. Cerebral dopamine neurotrophic factor improves long-term memory in APP/PS1 transgenic mice modeling Alzheimer's disease as well as in wild-type mice. Behav Brain Res. 2015;291:1-11.

23. Bäck S, Peränen J, Galli E, Pulkkila P, Lonka-Nevalaita L, Tamminen T, et al Gene therapy with AAV2-CDNF provides functional benefits in a rat model of Parkinson's disease. Brain Behav. 2013:3:75-88.

24. Choi JM, Hong JH, Chae MJ, Ngyuen PH, Kang HS, Ma HI, et al. Analysis of mutations and the association between polymorphisms in the cerebral dopamine neurotrophic factor (CDNF) gene and Parkinson disease. Neurosci Lett. 2011:493:97-101.

25. Lindholm D, Mäkelä J, Di Liberto V, Mudò G, Belluardo N, Eriksson O, et al. Current disease modifying approaches to treat Parkinson's disease. Cell Mol Life Sci. 2016;73(7):1365-79.

26. Voutilainen $M H$, Bäck S, Pörsti $E$, Toppinen $L$, Lindgren $L$, Lindholm $P$, et al. Mesencephalic astrocyte-derived neurotrophic factor is neurorestorative in rat model of Parkinson's disease. J Neurosci. 2009;29:9651-9.

27. Airavaara M, Harvey BK, Voutilainen $\mathrm{MH}$, Shen $\mathrm{H}$, Chou J, Lindholm $\mathrm{P}$, et al. CDNF protects the nigrostriatal dopamine system and promotes recovery after MPTP treatment in mice. Cell Transplant. 2012;21(6):1213-23.

28. Li W, Song $X$, Zhang $H$, Yang $Y$, Jiang $C$, Xiao B, et al. Association study of RELN polymorphisms with schizophrenia in Han Chinese population. Prog Neuropsychopharmacol Biol Psychiatry. 2011;35:1505-11.

29. Ross CA, Margolis RL, Reading SA, Pletnikov M, Coyle JT. Neurobiology of schizophrenia. Neuron. 2006;52:139-53.

30. Schizophrenia Psychiatric Genome-Wide Association Study (GWAS) Consortium. Genome-wide association study identifies five new schizophrenia loci. Nat Genet. 2011;43(10):969-76.

31. Li Z, Chen J, Yu H, He L, Xu Y, Zhang D, et al. Genome-wide association analysis identifies 30 new susceptibility loci for schizophrenia. Nat Genet. 2017:49(11):1576-83.

32. Muesser KT, McGurk SR. Schizophrenia. Lancet. 2004;363:2063-72.

33. Austin J. Schizophrenia: an update and review. J Genet Couns. 2005;14:329-40.

34. Carlsson A. The current status of the dopamine hypothesis of schizophrenia. Neuropsychopharmacology. 1988;1:179-86.

35. Abi-Dargham A. Schizophrenia: overview and dopamine dysfunction. J Clin Psychiatry. 2014;75:e31.

\section{Submit your next manuscript to BioMed Central and we will help you at every step:}

- We accept pre-submission inquiries

- Our selector tool helps you to find the most relevant journal

- We provide round the clock customer support

- Convenient online submission

- Thorough peer review

- Inclusion in PubMed and all major indexing services

- Maximum visibility for your research

Submit your manuscript at www.biomedcentral com/submit
C BioMed Central 